\title{
Drohnen als Partner im Luftraum
}

\author{
Rahmenbedingungen für eine Regulierung \\ des zivilen Drohnenwesens
}

\begin{abstract}
Markus Christen, Digital Society Initiative, Universität Zürich, Rämistrasse 66, 8001 Zürich (christen@ethik.uzh.ch), (1) orcid.org/0000-0001-8378-9366 Michel Guillaume, Zentrum für Aviatik, Zürcher Hochschule für Angewandte Wissenschaften (michel.guillaume@zhaw.ch) Maximilian Jablonowski, Institut für Sozialanthropologie und Empirische Kulturwissenschaft, Universität Zürich (jablonowski@isek.uzh.ch) Peter Lenhart, Zentrum für Aviatik, Zürcher Hochschule für Angewandte Wissenschaften (peter.lenhart@zhaw.ch), (1) orcid.org/orcid.org/0000-0002-4566-4754 Kurt Moll, Rechtsanwalt, Bern (mail@kurtmoll.ch)
\end{abstract}

In unserem Beitrag fassen wir drei Themen einer jüngst veröffentlichten Studie zur Technikfolgenabschätzung ziviler Drohnen zusammen. Erstens legen wir dar, dass der Rechtsbegriff der Drohne unklar geregelt ist und dass eine technische Definition des Begriffs „Drohne“ der falsche Weg ist. Wir präsentieren eine alternative Einbindung des Drohnenbegriffs in das europäische Luftfahrtrecht. Zweitens beschreiben wir die Komplexität der technisch zu regelnden Aspekte, um eine Einbindung von Drohnen in das Luftraumsystem ohne direkten Sichtkontakt zu ermöglichen. Wir beschreiben das Problem der technischen Sicherheit und die Komponenten eines Verkehrsmanagementsystems für Drohnen. Drittens verdeutlichen wir, dass bei der Bewältigung der ethischen, rechtlichen und sozialen Herausforderungen von Drohnen eine vollzugslücke besteht, insbesondere im Datenschutz.

\section{Drones as partners in the airspace}

Framework conditions for regulation of civilian drones

In our article, we summarize three topics of a recently published study on technology assessment of civilian drones. First, we explain that the legal concept of drones is unclear and that a technical definition of the term "drone" would be the wrong way to go. We present an alternative approach to integrate the concept of drones into European aviation law. Second, we describe the complexity of the technical aspects to be regulated in order to enable the integration of drones into the airspace system beyond visual line of sight. We describe the problem of technical safety and the components of a drone traffic management system. Third, we point to an enforcement gap in coping with the ethical, legal, and social challenges of drones, particularly in data protection.

Keywords: drone regulation; U-Space; drone safety; data protection

This is an article distributed under the terms of the Creative Commons Attribution License CCBY 4.0 (https://creativecommons.org/licenses/by/4.0/)

https://doi.org/10.14512/tatup.27.3.20

Submitted: 17. 07.2018. Peer reviewed. Accepted: 08.10.2018

\section{Einführung}

Ursprünglich für militärische Zwecke entwickelt, werden Drohnen heute verstärkt zivil verwendet: für Luftaufnahmen, Überwachung von Infrastruktur, Landwirtschaft, humanitäre Hilfe, Freizeit und vieles mehr. Die rasante Entwicklung des Mobilfunks und die Forschung zur Miniaturisierung und Autonomisierung von Drohnen geben dieser Technologie ein großes Zukunftspotenzial. Allerdings sind heute zentrale technische, regulatorische und soziale Fragen ungeklärt; diese bilden die Voraussetzung für eine verantwortungsbewusste Entwicklung des Drohnenwesens in Europa. Dies betrifft unter anderem die rechtliche Einordnung von Drohnen, ihre Einbindung in den Luftraum, wenn sie außerhalb des Sichtbereichs des Piloten fliegen sowie die Durchsetzung geltenden Rechts, das durch die zivile Nutzung von Drohnen tangiert wird.

Unser Beitrag beruht auf einer jüngst in der Schweiz veröffentlichten Studie zur Technikfolgenabschätzung ziviler Drohnen (Christen et al. 2018). Diese Studie legt Chancen und Risiken der Drohnentechnologie dar und zeigt ihr Entwicklungspotenzial in Forschung und Wirtschaft auf. Hier werden wir drei Aspekte genauer beleuchten. Erstens weisen wir auf den international unklaren Rechtsbegriff „Drohnen“ und damit zusammenhängende Abgrenzungsprobleme hin. Zweitens legen wir dar, dass eine erfolgreiche Nutzung der Drohnentechnologie eine klar definierte Einbindung in den Luftraum erforderlich macht. Drittens verdeutlichen wir, dass für viele anwendungsbezogene Probleme von Drohnen keine materiellen (inhaltlichen) Gesetzeslücken bestehen, sondern es am Vollzug bestehender Gesetze mangelt. Im Fazit fassen wir die wichtigsten Rahmenbedingungen für eine Regulierung des Drohnenwesens zusammen. Der Fokus liegt auf der kommerziellen Nutzung ziviler Drohnen; die rechtliche Analyse stützt sich maßgebend auf die Rechtslage in der Schweiz. 


\section{Der Rechtsbegriff „Drohnen“}

Der Begriff „Drohne“ ist die umgangssprachliche Bezeichnung für (bestimmte) unbemannte Luftfahrzeuge (Unmanned Aircraft, UA). In der wissenschaftlichen Literatur existieren zahlreiche Bezeichnungen für Drohnen (Clarke 2014), es besteht derzeit aber keine einheitliche und insbesondere rechtlich verbindliche Definition von Drohnen. Die begriffliche Unschärfe verdeutlicht, dass die Drohnentechnologie noch in der Entwicklung ist und dass der Begriff Drohne Systeme mit unterschiedlichem Erscheinungsbild bezeichnet.

Zudem werden Modellluftfahrzeuge schon seit vielen Jahrzehnten als UA genutzt. Grob kann man Modellluftfahrzeuge als Fluggeräte bezeichnen, die in verkleinerter oder miniaturisierter Weise meist ein Vorbild aus der bemannten Luftfahrt abbilden und meist zum Zweck des Vergnügens oder der Freizeitgestaltung betrieben werden. Es existiert aber keine klare Abgrenzung zwischen Drohnen und Modellluftfahrzeugen, was für Modellluftfahrzeugnutzer problematisch werden könnte. Diese befürchten, dass eine Regulierung von (professionellen) Drohnen die Nutzung von Modellluftfahrzeugen für Hobbyzwecke einschränkten könnte (Germann 2016, S. 13).

Diese begriffliche Unschärfe ist nicht nur bezüglich einer Abgrenzung zwischen Drohnen und Flugmodellen ein Problem. Generell braucht eine rechtliche Analyse und Systematisierung von Sachverhalten, in denen zivile Drohnen eingesetzt werden, eine klare Begriffsdefinition. Es sollte bestimmbar sein, wann es sich bei einem Fluggerät um eine Drohne im Rechtssinne handelt und wann nicht. Eine Definition sollte zudem genügend offen sein, um zu erwartende technische Entwicklungen einzubinden, damit die Regulation nicht der Innovation hinterherhinkt. the safe conduct of the aircraft throughout its flight." (ICAO 2011, S. 7)

Diese Definition enthält die Voraussetzung, dass das System durch einen lizenzierten Piloten kontrolliert wird, der zwar außerhalb des RPA situiert ist, jedoch zu jeder Zeit für die Flugsicherung ansprechbar und verantwortlich ist. Der Begriff Drohne wird im Rundschreiben nicht explizit erwähnt (Abbildung 1 , unten links).

Die Luftfahrtbehörde European Aviation Safety Agency (EASA) wurde von der Europäischen Kommission im Jahr 2014 beauftragt, ein Regulierungskonzept im Bereich Drohnen zu erarbeiten. Bereits 2015 wurden vorläufige Ergebnisse vorgestellt (EASA 2015). Die Schwierigkeiten im Zusammenhang mit der Definition des Begriffs Drohne wurden bereits von Beginn an thematisiert (EASA 2015, S. 2). Schließlich veröffentlichte die EASA die Prototype Commission Regulation on Unmanned Aircraft Operations vom 22. August 2016 (EASA 2016). Der Begriff Drohne fehlt im vorgeschlagenen Regulierungsvorschlag, er taucht aber in der Explanatory Note zum Regulierungsvorschlag an verschiedenen Stellen explizit auf, woraus ersichtlich wird, dass der vorgeschlagene Entwurf im Wesentlichen auf Drohnen zugeschnitten ist. Im Vorschlag wird ausschließlich von ,unmanned aircraft" als Grundbegriff ausgegangen. Darunter soll jedes Luftfahrzeug (aircraft) fallen, das ohne Pilot an Bord betrieben wird. Diese Definition hat heftige Irritationen ausgelöst, weil keinerlei Abgrenzungen zu Modellluftfahrzeugen vorgesehen sind (Abbildung 1, unten rechts).

Beispielhaft für diese Debatten ist das Schweizer Recht. Hier lassen sich zwei Ansätze zur begrifflichen Fassung von Drohnen unterscheiden, insbesondere bezüglich ihrer Abgrenzung von Flugmodellen: Entweder sind Drohnen vollständig in der Kategorie der Modellluftfahrzeuge enthalten (Abbildung 1, oben

\section{Eine rechtliche Analyse und Systematisierung von Sachverhalten, in denen zivile Drohnen eingesetzt werden, braucht eine klare Begriffsdefinition.}

Auf internationaler Ebene finden sich unterschiedliche Definitionen (Abbildung 1). In einem Rundschreiben der Internationalen Zivilluftfahrtorganisation (ICAO) zu unbemannten Luftfahrzeugsystemen von 2011 tauchen die Begriffe ,Flugmodelle“ (model aircraft), ,RPAS“ (remotely piloted aircraft system) und ,autonome Fluggeräte“ (autonomous aircraft) auf. Die ICAO definiert den Begriff RPA als

, ,...] aircraft piloted by a licensed "remote pilot" situated at a "remote pilot station" located external to the aircraft (i. e. ground, ship, another aircraft, space) who monitors the aircraft at all times and can respond to instructions issued by ATC, communicates via voice or data link as appropriate to the airspace or operation, and has direct responsibility for links), oder Drohnen und Modellluftfahrzeuge bilden zwei sich ausschließende Mengen (Abbildung 2, oben rechts). Für letzteren Ansatz wird vorgeschlagen, Drohnen über ihren Verwendungszweck zu definieren. Dies wäre ein falscher Weg, denn es kann nicht im Belieben des Nutzers liegen, wie ein bestimmtes Gerät rechtlich zu qualifizieren ist. Im Bereich der Modellflugzeuge und Drohnen ist nicht einsehbar, weshalb einzelne Modellluftfahrzeuge nicht die gleichen Funktionalitäten sollen ausüben können wie Drohnen, auch wenn es tatsächliche Unterschiede bei einem Einsatz geben mag (wie z. B. Reichweite, Geschwindigkeit, Einsatzdauer). Jeder Versuch einer Definition müsste sich an objektiven Eigenschaften einer Drohne orientieren. Bisher ist keine derartige Definition bekannt, was insbe- 
sondere an der Vielfalt der Drohnentypen liegt. Es erscheint unwahrscheinlich, dass eine technische Definition Drohnen klar von Flugmodellen abgrenzen kann.

Wir sind zu dem Schluss gekommen, dass eine begriffliche Unterscheidung zwischen Modellluftfahrzeugen und Drohnen technisch nicht exakt gezogen werden kann, aber auch nicht zwingend notwendig erscheint, weil unterschiedliche Regulierungsfolgen (nicht aber die Definition als solche) über verschiedene Einsatzzwecke definiert werden könnten. Störend ist allerdings, dass der Begriff Modellluftfahrzeug in der heutigen Gesetzgebung dominiert. In der Schweiz erscheint er als gesetzlicher Oberbegriff, was bereits rein etymologisch nicht überzeugt: Der Begriff Modellluftfahrzeug deutet darauf hin, dass es sich um eine Miniaturisierung eines größeren Originals (z. B. Flugzeug oder Helikopter) handelt, was bei Drohnen in der Regel gerade nicht der Fall ist. Das deutsche Luftverkehrsgesetz nennt den Begriff Drohne ebenfalls nicht, unterscheidet aber bei den unbemannten Fluggeräten zwischen solchen, die nicht zu Zwecken des Sports oder der Freizeitgestaltung betrieben werden (i. e. unbemannte Luftfahrtsysteme) und Flugmodellen (BJV 2017, § 1). Der Begriff „unbemannte Luftfahrtsysteme“ zur Bezeichnung kommerziell genutzter Drohnen erscheint allerdings ebenfalls ungeeignet, weil er international als Überbegriff für alle unbemannten Fluggeräte verwendet wird. Es sollte einen geeigneten Überbegriff über die Unterkategorien Flugmodelle und Drohnen geben.

\section{Einbindung in den Luftraum}

\section{Kontrollierter und unkontrollierter Luftraum}

Der Luftraum ist gemäß Vorgaben der ICAO in verschiedene Zonen unterteilt. Grundsätzlich unterscheidet man zwischen dem kontrollierten und dem unkontrollierten Luftraum. Im kontrollierten Luftraum - in der Regel die Zone ab 2000 Fuß $(610 \mathrm{~m})$ über Grund - wird der Flugverkehr von der Flugsicherung überwacht. Der kontrollierte Luftraum besteht aus mehreren Luftraumklassen: Je nach Klasse wird der Luftverkehr gestaffelt, d.h. der Fluglotse ist in seinem Sektor dafür verantwortlich, die Flugzeuge so zu lotsen, dass es nicht zu gefährlichen Annährungen kommt.

Damit Drohnen ohne direkten Sichtkontakt zum Piloten im kontrollierten Luftraum betrieben werden können, müssen folgende Voraussetzungen erfüllt sein: Erstens muss die Drohne über die gleichen Bordsysteme wie jedes andere Verkehrsflugzeug verfügen (Autopilot, Radionavigation etc.). Zweitens muss der Drohnenpilot an der Bodenstation in der Lage sein, die gleichen Aufgaben zu erfüllen, wie wenn er an Bord des Flugzeugs wäre. Dazu zählen insbesondere die Kommunikation mit dem Fluglotsen und die Möglichkeit, Anweisungen des Fluglotsen als Steuerkommandos an die Drohne zu schicken. Drittens muss der Gesetzgeber die Drohne von der Pflicht zum Sehen und Gesehenwerden (see and avoid) unter der Voraussetzung befreien, dass die Staffelung durch die Flugsicherung und das Airborne Collision Avoidance System genügend Kollisionssicherheit bieten. In der Praxis verfügen nur entsprechend große Drohnen über die Zuladungskapazität für hierzu notwendige Bordelektronik.

Die meisten Drohnen fliegen heute im unkontrollierten Luftraum, wo der Flugverkehr nicht von der Flugsicherung überwacht und gestaffelt wird. Auch wenn Instrumentenflug (Pilotieren ohne optisch wahrnehmbare Referenzpunkte) dort nicht verboten ist, müssen von allen Luftverkehrsteilnehmern die Ausweichregeln des Sichtflugs angewendet werden. Es gilt das Prinzip „Sehen und Gesehenwerden“. Betreiber von Drohnen gehen oft davon aus, dass im untersten Höhenband des unkontrollierten Luftraums (abgesehen von Flugplätzen) - also bis $2000 \mathrm{Fuß}$ über Grund - keine Luftfahrt stattfindet. Diese Annahme ist falsch. Zwar sind Piloten generell dazu angehalten, außer zu Start und Landung in mindestens 500 Fuß $(152 \mathrm{~m})$ über Grund zu fliegen. In der Praxis geht dies jedoch nicht immer. Beispiele sind Ballonfahrer, Gleitschirmpiloten und Segelflieger, die auf einem Acker landen müssen, oder Rettungshubschrauber, die außerhalb von Flugplätzen und Heliports landen und starten. Zudem werden Helikopter zum Personen- und Materialtransport eingesetzt. Drohnen, die im unkontrollierten Luftraum betrieben werden sollen, müssen also in der Lage sein, das Prinzip „Sehen und Gesehenwerden“ uneingeschränkt zu erfüllen. 
Genau dies ist das Kernproblem der heutigen Nutzung von Drohnen. Die Sichtflugregeln beinhalten Vorschriften, die festlegen, wie die Luftfahrzeuge einander ausweichen müssen - ähnlich den Vorfahrtsregeln im Straßenverkehr. Das Prinzip ,Sehen und Gesehenwerden“ bedingt gemäß dem in der Luftfahrt gängigen Verständnis ausgereifte kognitive Fähigkeiten, denn die Befolgung dieses Prinzips ist nicht nur eine Frage der Wahrnehmung, sondern auch des Erkennens potenzieller Absichten des Gegenübers. Technisch realisierte Autonomiefähigkeit dürfte zwar bald zu Kollisionsschutzsystemen führen, die über elektronische Sensoren die anderen Luftverkehrsteilnehmer rechtzeitig erkennen und bei Bedarf Manöver einleiten können (detect
Ein nicht zu unterschätzendes Risiko bilden deshalb Kollisionen mit anderen Luftraumteilnehmern. Mit der zunehmenden Verbreitung von Drohnen stellen diese zum Beispiel bei Einsätzen von Rettungskräften eine große Gefahr dar, wenn Rettungshelikopter oder Löschflugzeuge behindert werden. In den letzten Jahren gab es regelmäßig Berichte von Beinahe-Kollisionen von Flugzeugen mit Drohnen. Für den Piloten eines Flugzeugs ist eine Drohne aus dem Cockpit auch mit Kenntnis der Position der Drohne enorm schwierig zu erkennen. Kontrollierte Versuche (Remote Vision 2016), in denen Annäherungsszenarien zwischen einer Drohne und einem Motorflugzeug getestet wurden und der Pilot stets Kenntnis über die Position der

\section{Die meisten Drohnen fliegen heute im unkontrollierten Luftraum, wo der Flugverkehr nicht von der Flugsicherung überwacht und gestaffelt wird.}

and avoid) - doch dies entspricht nicht dem See-and-avoid-Prinzip. Genau aus diesem Grund ist in der Schweiz und anderswo ein direkter Sichtkontakt mit der Drohne obligatorisch, um diese betreiben zu können (für Ausnahmen braucht es Spezialbewilligungen). Der Drohnenpilot muss also während des gesamten Fluges Blickkontakt zu seinem Fluggerät haben, das Fluggerät muss in visual line of sight (VLOS) sein. Dies bedingt insbesondere, dass der Drohnenpilot Fluglage und Flugrichtung der Drohne jederzeit eindeutig erkennen können muss, um einen sicheren Flugbetrieb zu gewährleisten. Selbst bei optimalen Sichtverhältnissen ist dieser Operationsraum sehr begrenzt. Bei einer Standard-Drohne (DJI Mavic mit 85 Millimeter Querschnitt) beträgt die maximale Entfernung, in der die Drohne noch sicher erkannt werden kann, lediglich 97 Meter (Christen et al. 2018). Viele der kommerziell interessanten Anwendungen sind also im VLOS-Betrieb nur sehr eingeschränkt möglich.

\section{Drohnen als Sicherheitsrisiko}

Die Erfordernisse der Flugsicherheit sind in mehrfacher Hinsicht ein Problem für die gegenwärtige Nutzung von Drohnen. Beim Flug im VLOS-Betrieb ist der Drohnenpilot für eine sichere Flugdurchführung verantwortlich. Er muss drohende Kollisionen rechtzeitig erkennen und angemessen darauf reagieren. Er erfüllt hier also die gleiche Aufgabe wie der Pilot eines Flugzeugs beim Sichtflug. Flugzeugbesatzungen müssen sich einer regelmäßigen flugmedizinischen Untersuchung unterziehen, bei der insbesondere die Sehfähigkeit geprüft wird. Es ist zu überdenken, ob entsprechende Auflagen nicht auch für Drohnenpiloten eingeführt werden müssen. Insbesondere bei Fluggeräten, bei denen der Autopilot die Lageregelung übernimmt, darf davon ausgegangen werden, dass der Drohnenpilot unter Umständen auch dann noch Sichtkontakt deklariert, wenn das Fluggerät für ihn nur noch als Punkt erkennbar ist.
Drohne hatte, haben gezeigt, dass bei der optischen Identifizierung einer Drohne meist nur knapp vier Sekunden für ein Ausweichmanöver übrigbleiben. Ein Motorflugpilot kann also eine Drohne in der Regel nicht erkennen und die Verantwortung für ein Ausweichmanöver muss beim Drohnenpiloten liegen. Dies wurde auch in den neusten Regeln der EASA (2017) entsprechend festgehalten.

Manntragende Luftfahrzeuge müssen entsprechend den heute geltenden Zertifizierungsgrundlagen nur Vorschriften für Kollisionen mit Vögeln erfüllen. Die Kollision eines Luftfahrzeugs mit einer Drohne ist jedoch selbst bei ähnlichem Gewicht nicht mit Vogelschlag vergleichbar (Hambling 2016). Eine Droh-

\section{Kollisionen mit anderen Luftraum- teilnehmern sind ein nicht zu} unterschätzendes Risiko. Auch die

\section{Sicherheit von Passanten und Tieren muss gewährleistet sein.}

ne besteht aus festen Materialien, die im Fall eines Einsaugens in ein Triebwerk oder beim Einschlag in eine Cockpitscheibe einen weitaus größeren Schaden anrichten können (bis hin zur vollständigen Zerstörung des Triebwerks oder Tötung des Piloten).

Beim Einsatz von Drohnen muss auch die Sicherheit von Passanten und Tieren gewährleistet sein. Zu Unfällen kann es kommen, wenn die Drohne über Personen oder Tieren unkontrolliert 
abstürzt oder wenn Start- oder Landezonen nicht vor einem unbefugten Zutritt gesichert sind. Dies ist insbesondere bei der geplanten Paketzustellung an private Lieferadressen eine Herausforderung. Ein außer Kontrolle geratener Multikopter kann ein erhebliches Gefährdungsrisiko darstellen (DEKRA 2017). Trotz des obligatorischen Meldewesens nach EU-Verordnung 376/2014 ist die Anzahl der Abstürze von Drohnen nahezu unbekannt, die Dunkelziffer dürfte enorm groß sein. Einzig in Bezug auf die militärische Verwendung von Drohnen sind ansatzweise Zahlen bekannt. Hier zeigt sich, dass die Ausfallraten bei Drohnen deutlich über jenen von manntragenden Flugzeugen lagen (Referenzen in Christen et al. 2018).

Zudem stellen Witterungseinflüsse wie Regen, Schnee, Eis, Feuchtigkeit, extreme Temperaturen, starke Winde sowie Turbulenzen Herausforderungen für die Strukturfestigkeit von Drohnen dar. Solche Aspekte müssen bei einer Risikound-Safety-Analyse berücksichtigt werden. Da Drohnen in tiefen atmosphärischen Schichten betrieben werden, dürfte das Problem der Vereisung eine besondere Herausforderung darstellen. Sollten dereinst Drohnen in rauen Wetterbedingungen eingesetzt werden, ist ein beträchtlicher Aufwand zu leisten, um zum Beispiel Redundanzen für die elektrische Versorgung und Navigation sowie die elektromagnetische Verträglichkeit und generell die Widerstandsfähigkeit gegen natürliche Einflussfaktoren wie Wasser oder Blitzeinschläge einzuführen (Referenzen in Christen et al. 2018).

\section{Verkehrsmanagementsystem für Drohnen}

Eine breite kommerzielle Nutzung von Drohnen dürfte nur dann gelingen, wenn Flüge ohne direkten Sichtkontakt (beyond visual line of sight, BVLOS) möglich werden, was die Lösung zahlreicher Sicherheitsfragen voraussetzt. Insbesondere braucht es ein brauchbares Kollisionsschutzsystem. Wenn Drohnen im Luftraum genutzt werden, wird man deshalb ein angepasstes Verkehrsmanagementsystem für Drohnen entwickeln müssen. In Europa spricht man diesbezüglich vom „U-Space“, definiert als "set of new services, and specific procedures designed to support safe, efficient and secure access to airspace for large numbers of drones" (SESAR JU 2017).

So ein System muss schrittweise aufgebaut werden, da neue Technologien und Verfahren eingesetzt werden. Der erste Schritt könnte die Einführung eines Drohnenkorridors im Luftraum unter 150 Meter sein. Danach könnte die Einführung kooperativer (d. h. entsprechend ausgerüstete Fluggeräte tauschen ihre Positionsdaten aus) und nicht-kooperativer Navigationssysteme in entsprechenden Flugzonen folgen. Ein dritter Schritt wäre dann die volle Integration der Drohnen ins heutige Luftfahrt System mit Instrumented-Flight-Rules-Operationen. Abbildung 2 zeigt die dafür notwendigen Elemente in einer Übersicht.

\section{Konkrete Regulation und Vollzug}

\section{Stand der internationalen Debatte}

Das internationale Regelwerk im Zusammenhang mit zivilen Drohnen ist unübersichtlich. Dies liegt einerseits daran, dass der rechtliche Status wesentlicher Akteure wie beispielsweise der Joint Authorities for Rulemaking on Unmanned Systems (JARUS) nicht vollständig geklärt und transparent ist. Der EASA fehlte auf der anderen Seite lange Zeit noch die Kompetenz, im Bereich der Drohnen unter $150 \mathrm{~kg}$ regulatorisch überhaupt Vorschläge machen zu können, da für die Regulierung und Zulassung von Flugobjekten unterhalb von 150 Kilogramm die nationalen Behörden zuständig sind.

Die EASA hat im August 2016 die inhaltliche Stoßrichtung ihres Regulierungsvorschlags präsentiert. Sie schlägt drei unterschiedliche Risikokategorien (Open - Specific - Certified) vor, die je eigene Regulierungserfordernisse aufweisen und die von JARUS übernommen wurden. Wesentliche Grundlage für die verschiedenen Risikobetrachtungen bildete die Zuordnung kinetischer Energien im Zusammenhang mit dem Betrieb ziviler Drohnen zur Wahrscheinlichkeit von Todesfolgen für Personen (nur im Bereich Open Category). Je nach Risikokategorie und Unter- 
kategorie sind verschiedene Maßnahmen (wie z. B. Geräte- und Operationslimitierungen, Typenprüfungen nach CE-Richtlinien), Geofencing, elektronische Registrierung, Pilotenanforderungen, Zertifizierungen) vorgesehen bzw. notwendig. Aufgrund zahlreicher offener Fragen und Kritikpunkte am EASA-Vorschlag war der europäische Regulierungsprozess in jüngster Zeit ins Stocken geraten. Insbesondere Modellflieger (aber nicht nur diese) bezweifeln die Angemessenheit verschiedener Drohnen-Regeln, die auch auf die von der EASA begrifflich nicht unterschiedenen Modellluftfahrzeuge anzuwenden wären. Im Juli 2018 haben nun EU-Parlament und Rat den Text einer neuen Verordnung zur Festlegung gemeinsamer Vorschriften für die Zivilluftfahrt beschlossen (EU 2018), welche auch die Schaffung von rechtlichen Grundlagen für die sichere Integration unbemannter Luftfahrzeuge in den europäischen Luftraum verlangt. Die EASA arbeitet aktuell an einer Konkretisierung dieses Beschlusses.

\section{Datenschutz}

Der Datenschutz im Zusammenhang mit den Einsatzmöglichkeiten ziviler Drohnen ist international ein breit diskutiertes Thema. Auch gemäß Medienanalyse und Expertenumfrage unserer Studie (Christen et al. 2018) ist die mögliche Verletzung der Privatsphäre durch Drohnen das für die Öffentlichkeit relevanteste Thema. Gestützt auf die Richtlinie des europäischen Parlaments und des Rates zum Schutz natürlicher Personen bei der Verarbeitung personenbezogener Daten und zum freien Datenverkehr (EPuR 1995, Art. 29) wurde auf europäischer Ebene eine Datenschutzgruppe eingesetzt. Diese hat im Zusammenhang mit zivilen Drohnen eine Stellungnahme veröffentlicht (Data Protection Working Party 2015). Darin werden verschiedene Forderungen an die Adresse der nationalen und auch des europäischen Gesetzgebers sowie an die Adresse von Hersteller und auch Betreibern formuliert, um die Datenschutzrisiken im Zusammenhang mit zivilen Drohnen beherrschbar zu halten.

Grundsätzlich lässt sich aber bezüglich der Gesetzeslage in der EU (und der Schweiz) feststellen, dass die Risiken der aktuellen Drohnennutzung bezüglich Privatsphäre überschaubar erscheinen und die materiellen Schutzbestimmungen ausreichen insbesondere auch mit Blick auf die neue Datenschutz-Grundverordnung der Europäischen Union. Diese stärkt das Recht auf informationelle Selbstbestimmung - doch insbesondere mit Kameras ausgerüstete Freizeitdrohnen können die faktische Wahrnehmung dieses Rechts erschweren. Die Rechtsprechung, die wissenschaftliche Lehre sowie weitere Stakeholder (Behörden, Legislative, NGOs) sind aber laufend damit beschäftigt, den Umfang und die Grenzen eines informationellen Selbstbestimmungsrechts auszuloten und weiterzuentwickeln. Diese Bestrebungen sind bereichsübergreifend und unabhängig von der Nutzung ziviler Drohnen, können und müssen aber selbstverständlich in diesem Bereich ebenfalls anhand konkreter Fragestellungen weitergeführt werden.

Mängel bestehen allerdings bei der formellen Durchsetzung der inhaltlichen Rechtsansprüche. Obwohl zivile Drohnen funktional meist nicht mehr sind als Kameras und andere bereits be- stehende Überwachungsinstrumente (auch wenn Drohnen bisweilen sehr viel effizienter agieren können), stehen der Durchsetzung materieller Schutzansprüche oft unlösbare Hindernisse gegenüber. Bei anonymen Einsätzen nicht registrierter Drohnen wird es einer in ihrer Privatsphäre oder in ihren datenschutzrechtlichen Ansprüchen verletzten Person in der Regel unmöglich sein, wirksam dagegen vorzugehen. Diese Situation ist aus rechtsstaatlicher Sicher unbefriedigend und nur mittels vorbeugender Schutzmaßnahmen (wie z. B. einem Drohnenregister) lösbar. Der Prüfung der Machbarkeit bzw. der Ausgestaltung einer solchen Maßnahme muss dabei Priorität zukommen, schließlich sollte der administrative Aufwand (insbesondere im Zusammenhang mit kleinen Drohnen) in einem vernünftigen Verhältnis zum damit verfolgten Zweck stehen.

\section{Fazit}

Die Einbindung von zivilen Drohnen in den Luftraum ist mit zahlreichen Herausforderungen verbunden, die nach Lösungen verlangen. Gemäß unserer Ansicht sind hierfür insbesondere folgende Maßnahmen erforderlich:

- Der Begriff Drohne ist - ohne ihn technisch von den Modelluftfahrzeugen abzugrenzen - im Regulativ einzuführen. Es sollten unterschiedliche Regulierungen für Einsatzbereiche von Drohnen und Modellluftfahrzeuge dort vorgesehen werden, wo sie aufgrund tatsächlicher Verhältnisse gerechtfertigt erscheinen und nicht begrifflich begründet sind.

- Forschungen und Stakeholder-Dialoge, welche zur Schaffung eines Flugverkehrsmanagements für unbemannte Luftfahrzeuge (U-Space) beitragen, sind zu fördern.

- Um Vollzugsprobleme z. B. im Bereich Datenschutz zu lösen, ist eine Registrierungspflicht erforderlich, damit den materiellen Normen auch tatsächlich Beachtung verschafft werden kann. Zudem sollte ein solches System ein elektronisches call sign haben, damit eine Drohne immer identifiziert werden kann. Ergänzend sind Richtlinien für die Ausbildung von Drohnenpiloten zu bestimmen und umzusetzen. Die Hersteller bzw. Händler von Drohnen sollten verpflichtet werden, den Verkaufs- bzw. Versandpackungen die maßgeblichen Bestimmungen zur geltenden Rechtslage beizulegen.

\section{Literatur}

BJV - Bundesministerium der Justiz und für Verbraucherschutz (2017): Luftverkehrsgesetz in der Fassung der Bekanntmachung vom 10. Mai 2007, zuletzt geändert durch Art. 2 Abs. 11 G v. 20.7.2017. Online verfügbar unter https://www.gesetze-im-internet.de/luftvg/BJNR006810922.html, zuletzt geprüft am 15.10.2018.

Christen, Markus; Guillaume, Michel; Jablonowski, Maximilian; Lenhart, Peter; Moll, Kurt (2018): Zivile Drohnen. Herausforderungen und Perspektiven. Zürich: vdf Hochschulverlag. Online verfügbar unter www.ta-swiss.ch/ projekte-und-publikationen/informationsgesellschaft/zivile-drohnen, zuletzt geprüft am 29.11.2018. 
Clarke, Roger (2014): Understanding the drone epidemic. In: Computer Law \& Security Review 30, S. 230-246.

Data Protection Working Party (2015): Opinion 01/2015 on Privacy and Data Protection Issues relating to the Utilisation of Drones, adopted on 16 June 2015. Online verfügbar unter https://ec.europa.eu/justice/article-29/ documentation/opinion-recommendation/files/2015/wp231_en.pdf, zuletzt geprüft am 17.10.2018.

DEKRA (2017): Hohe Verletzungsgefahr durch Drohnen. Online verfügbar unter https://www.dekra.de/de-de/hohe-risiken-durch-kleine-flieger, zuletzt geprüft am 15.07.2018.

EASA - European Aviation Safety Agency (2015): Vorschlag für die Erstellung von gemeinsamen Vorschriften für den Betrieb von Drohnen in Europa. Online verfügbar unter https://www.easa.europa.eu/download/ANPAtranslations/205933_EASA_Summary\%20of\%20the\%20ANPA_DE.pdf, zuletzt geprüft am 15. 07.2018.

EASA (2016): „Prototype“ commission regulation on unmanned aircraft operations. Online verfügbar unter https://www.easa.europa.eu/sites/default/ files/dfu/UAS\%20Prototype\%20Regulation\%20final.pdf, zuletzt geprüft am 15. 07.2018.

EASA (2017): Introduction of a regulatory framework for the operation of drones. Notice of proposed amendment 2017-05 (A). Online verfügbar unter https://wpo-altertechnology.com/easa-european-aviation-safetyagency-notice-proposed-amendment-2017-05, zuletzt geprüft am 27.09.2018

EPUR - Europäisches Parlament und Rat (1995): Richtlinie 95/46/EG des Europäischen Parlaments und des Rates vom 24. Oktober 1995 zum Schutz natürlicher Personen bei der Verarbeitung personenbezogener Daten und zum freien Datenverkehr. Online verfügbar unter https://eur-lex.europa. eu/legal-content/DE/TXT/?uri=celex\%3A31995L0046, zuletzt geprüft am 15. 10.2018 .

EU - European Union (2018): Regulation (EU) 2018/1139 of the European Parliament and of the Council of 4 July 2018 on common rules in the field of civil aviation and establishing a European Union Aviation Safety Agency. Online verfügbar unter http://data.europa.eu/eli/reg/2018/1139/oj, zuletzt geprüft am 25.09.2018.

Germann, Peter (2016): Modellflug und Drohnenbetrieb. Der Unterschied. In: AeroRevue Heft 11/2016, S. 13.

Hambling, David (2016): What really happens when a drone strikes an airplane. Online verfügbar unter http://www.popularmechanics.com/flight/drones/ a24467/drone-plane-collision, zuletzt geprüft am 15. 07.2018.

ICAO - International Civil Aviation Organization (2011): Unmanned aircraft systems (UAS). Online verfügbar unter https://www.icao.int/Meetings/UAS/ Documents/Circular\%20328_en.pdf, zuletzt geprüft am 15.07.2018.

Remote Vision (2016): SVZD See and Avoid mit FLARM. Online verfügbar unter https://www.youtube.com/watch?v=pXIbra6P8zc, zuletzt geprüft am 15. 07.2018.

SESAR JU - Single European Sky Air traffic management Research Joint Undertaking (2017): U-space blueprint. Key principles of u-space. Luxemburg: Publications Office of the European Union. D0I: 10.2829/335092.

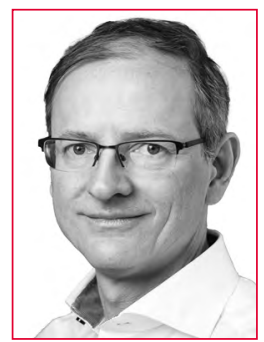

\section{PD DR. MARKUS CHRISTEN}

ist seit 2016 Geschäftsführer der Digital Society Initiative und leitet eine Forschungsgruppe am Institut für Biomedizinische Ethik der Universität Zürich. Seine Forschungsgebiete sind Ethik von Informations- und Kommunikationssystemen, Neuroethik und Empirische Ethik.

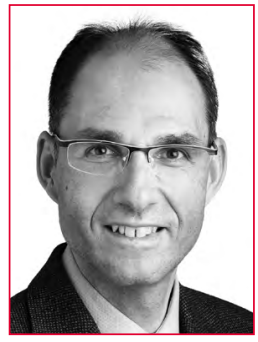

\section{PROF.DR. MICHEL GUILLAUME}

ist seit 2012 Leiter des Zentrums für Aviatik an der Zürcher Hochschule für Angewandte Wissenschaften und seit 2016 Professor für Systemintegration und Strukturintegrität. Er lehrt und forscht in Aerodynamik, Flugzeug-Strukturdesign sowie Systems Engineering in Unmanned Aviation und Space Systems.

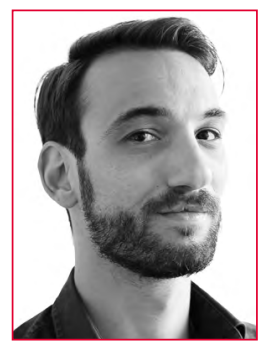

\section{MAXIMILIAN JABLONOWSKI}

ist seit 2013 Assistent am Institut für Sozialanthropologie und Empirische Kulturwissenschaft der Universität Zürich, wo er mit einer Arbeit über die soziotechnischen Imaginationen zu zivilen Drohnen doktorierte. Seine Forschungsschwerpunkte sind Science \& Technology Studies sowie Visual Anthropology.

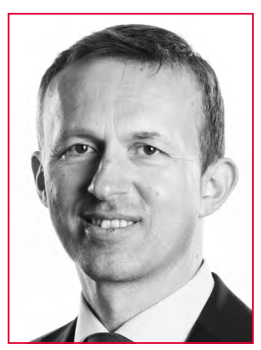

\section{DR. PETER M. LENHART}

ist seit 2014 Dozent an der Zürcher Hochschule für Angewandte Wissenschaften. Er leitet dort die Forschungsgruppe Mensch-Maschine-Systeme in der Luftfahrt. Seine Forschungsinteressen umfassen die Mensch-Drohne-Interaktion und Flugbetriebsverfahren für unbemannte Luftfahrzeuge.

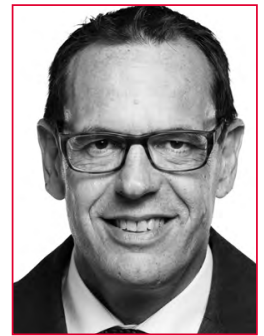

\section{DR. KURT MOLL}

ist seit 2006 selbständiger Rechtsanwalt in Bern und seit 2007 Dozent für Verkehrsrecht an der Zürcher Hochschule für angewandte Wissenschaften. Seine Forschungs- und Studienschwerpunkte liegen auf regulatorischen Zusammenhängen und Konzepten im Verkehrsbereich. 